\title{
The end of HIV: how do we get there?
}

\section{Treatment is now the focus of prevention}

T he world's HIV research policy and advocacy community will converge in Melbourne in July for the 20th International AIDS Conference. This is the first time this particular event has been held in this country, and provides an opportunity to focus on our response to the epidemic in our region, where we are now, and how to reduce transmission to zero. Over the years, the prevailing metaphor has evolved from the Grim Reaper's "prevention is the only cure we've got" to its polar opposite, "treatment as prevention" ${ }^{1,2}$ Recent high-profile reports of "cures" provide hope that ending HIV is a possibility..$^{3-5}$ But what is needed to ensure this goal is accessible to all?

While Australia's clinical and public health response to HIV has ensured that we remain a low-prevalence country with a relatively concentrated epidemic, there is currently a rising rate of new infections - the highest in more than 20 years. ${ }^{6}$ To meet this challenge, state and national HIV strategies have outlined targets that aim to halve sexual transmission by 2015, with the goal of virtual elimination of transmission by $2020{ }^{7,8}$ To achieve this, testing rates need to double and antiretroviral coverage needs to be around $95 \% .{ }^{7}$ Lowering the barriers to HIV testing, as well as the Pharmaceutical Benefits Advisory Committee's recent removal of the need for a $\mathrm{CD} 4$ criterion to prescribe subsidised antiretroviral treatment, are key steps towards a "test and treat" approach.

The dialogue with patients is changing; they are being encouraged to take responsibility not only for their own health but for that of others, by choosing to commence antiretroviral therapy at an earlier stage. The evidence for this "treatment as prevention" public health approach was first demonstrated by the HTPN 052 study, in which transmission was reduced by $96 \%$ in serodiscordant couples receiving early treatment, regardless of CD4 count. ${ }^{2}$ This approach is not the only answer, and the safe sex message that also applies to many other sexually transmissible infections should not be lost.

With the life expectancy of a person living with HIV approaching that of the general population, the focus now is on chronic disease management. Modelling predicts that the population living with HIV is not only ageing, but will increase in size due to increased life expectancy as well as the burden of new infections. While AIDS-defining illnesses are declining, significant contributors to morbidity and mortality are serious non-AIDS illnesses (SNAs), including malignancy and cardiovascular, cerebrovascular, liver and renal disease. It still remains to be seen whether the timing of antiretrovirals affects the individual risk of developing these complications - the Strategic Timing of Antiretroviral Treatment (START) study, due for completion in December 2015, aims to answer this question.

The rollout of antiretrovirals in the developing world has ensured that 10 million people now have access to treatment, thanks to programs such as PEPFAR and the Global Fund to Fight AIDS, Tuberculosis and Malaria. As these funding sources decline, vulnerable groups need to be the

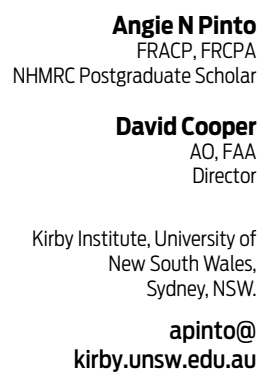

doi: 10.5694/mjal4.00682 
focus of locally based programs to ensure the continuum of care: from identifying those infected to being diagnosed, engaged in care, retained in care and, finally, initiated and maintained on treatment. Australia should continue its commitment to support countries in our region with concentrated epidemics in vulnerable populations such as Papua New Guinea, Cambodia, Indonesia and Myanmar. Priorities are to ensure access to treatment, reduce detectable viral load and ultimately stop HIV transmission.

Despite the success of antiretroviral therapy, it is not curative, and there is an ongoing role for vaccine development and research into other novel therapies. Progress has also been made in vaccine development, with modest evidence for prevention of HIV being demonstrated for the first time in the RV 144 Thai vaccine trial. The T cell vaccine approach is also being explored, alone and in combination with neutralising antibody vaccines. Cell therapies such as transcriptional gene silencing could facilitate viral latency, resulting in functional cure. Gene therapies that prevent HIV binding to and entering cells may also be the source of breakthroughs in the future.

An HIV-free generation is closer than ever before, and can be achieved through targeted campaigns, enhanced testing and treatment rollout. Simultaneously, ongoing research into vaccines and potential cures must continue, as chronic disease management is not the end goal.

Competing interests: No relevant disclosures.

Provenance: Commissioned; externally peer reviewed.

1 Reynolds S. Grim Reaper [video]. https://www.youtube.com/ watch?v=U219eUIZ7Qo (accessed Jun 2014).

2 Cohen MS, Chen YQ, McCauley M, et al; HPTN 052 Study Team. Prevention of HIV-1 infection with early antiretroviral therapy. N Engl J Med 2011; 365: 493-505.

3 Lisziewicz J, Rosenberg E, Lieberman J, et al. Control of HIV despite the discontinuation of antiretroviral therapy. NEngl J Med 1999; 340: 1683-1684.

4 Hütter G, Nowak D, Mossner M, et al. Long-term control of HIV by CCR5 Delta32/Delta32 stem-cell transplantation. N Engl J Med 2009; 360: 692-698.

5 Persaud D, Gay H, Ziemniak C, et al. Absence of detectable HIV-1 viremia after treatment cessation in an infant. N Engl J Med 2013; 369: 1828-1835.

6 Kirby Institute. HIV, viral hepatitis and sexually transmissible infections in Australia: annual surveillance report 2013. Sydney: Kirby Institute, 2013. http://www.kirby.unsw.edu.au/surveillance/2013-annual-surveillancereport-hiv-viral-hepatitis-stis (accessed Jun 2014).

7 NSW Ministry of Health. NSW HIV Strategy 2012-2015: a new era. http:// www.health.nsw.gov.au/publications/Pages/nsw-hiv-strategy-2012-15-anew-era.aspx (accessed Jun 2014).

8 Australian Government Department of Health. Consultation draft. Seventh national HIV strategy 2014-2017. https: //consultations.health. gov.au/ohpd-health-protection-policy-branch/test-national-strategiesfor-blood-borne-viruses-a/supporting documents/Draft $\% 207$ th $\% 20$ National\%20HIV\%20Strategy\%2020142017\%20D14818811.PDF (accessed Apr 2014). 